\title{
OBTENCIÓN DE LÍNEAS F 4 DE AMARANTO (Amaranthus spp.) A PARTIR DE CRUZÂMIENTOS INTERVARIETALES
}

\section{DEVELOPMENT OF F LINES OF AMARANTH (Amaranthus spp.) FROM INTERVARIETAL CROSSES}

\author{
Leticia Tavitas-Fuentes ${ }^{1}$, Leonardo Hernández-Aragón ${ }^{1}$, Micaela de la 0-Olan ${ }^{2}$, Alfredo \\ Josué Gámez-Vázquez ${ }^{3}$, Dora Ma. Sangermán-Jarquín ${ }^{2}$ y José Luis Arellano-Vázquez²
}

\footnotetext{
'Instituto Nacional de Investigaciones Forestales, Agrícolas y Pecuarias (INIFAP), Campo Experimental Zacatepec, Zacatepec, Morelos, México. ${ }^{2}$ INIFAP, Campo Experimental Valle de México, Texcoco, Estado de México, México. ${ }^{3}$ NIFAP, Campo Experimental Bajío, Celaya, Guanajuato, México.

*Autor de correspondencia (micaelaolan@yahoo.com.mx)
}

\section{RESUMEN}

En el cultivo de amaranto (Amaranthus spp.) es importante desarrollar nuevas variedades con el ideotipo requerido por los productores, aptas para siembra y cosecha mecanizada, con porte intermedio para que resistan el acame, uniformidad en altura de planta, longitud de inflorescencia, maduración del grano, resistencia a enfermedades y al desgrane, alto potencial de rendimiento y buena calidad del grano. En México, el mejoramiento genético del género Amaranthus está sólo basado en selección masal, obteniendo materiales a través del fenotipo y no se ha explotado completamente la diversidad genética del cultivo; por lo anterior, el objetivo del presente estudio fue obtener líneas avanzadas $\mathrm{F}_{4}$ a través de cruzamientos simples para avanzar a una fase superior y contar con líneas promisorias y variedades adecuadas para los productores de amaranto. Con la técnica del cultivo de mini-plantas se realizaron cruzas dirigidas de las que se obtuvieron 6404 semillas $F_{2}$, con las que se constituyeron grupos uniformes. En el ciclo primavera-verano (PV)-2017 se seleccionaron por el método masivo modificado 18 poblaciones segregantes $\mathrm{F}_{3}$ y en el PV-2018 se continuó con el mismo método donde se obtuvieron ocho poblaciones segregantes $\mathrm{F}_{4}$ con plantas de 1.30 a $1.65 \mathrm{~m}$ de altura, aptas para la cosecha mecanizada, panojas compactas, con mayor sincronía en la floración, lo que se tradujo en un mayor peso de grano por panoja ( $80 \%$ ). A través de la mejora de amaranto por recombinación genética es factible la generación de variedades modernas aptas para la cosecha mecanizada, con rentabilidad y sustentabilidad del cultivo.

Palabras clave: Amaranthus spp., cruzamientos intervarietales, mejoramiento genético.

\section{SUMMARY}

In the amaranth crop (Amarantus spp.) it is important to develop new varieties with the ideotype required by producers, suitable for mechanized planting and harvesting, with medium plant height to resist lodging, uniformity in plant height, inflorescence length, grain maturation, resistance to diseases and shattering, high yield potential and and good grain quality. In Mexico, the amaranth breeding is only based on mass selection, obtaining materials through the phenotype, and genetic diversity of the crop has not been fully exploited; therefore, the objective of this study was to obtain advanced $F_{4}$ lines through single crosses to advance to a higher phase and have promising lines and suitable varieties for amaranth producers. With the mini-plant cultivation technique, direct crosses were made, from which 6404 seeds in $F_{2}$ were obtained, and those were used to constitute uniform groups. During the
Spring-Summer (SS)-2017 agricultural cycle, 18 segregating $F_{3}$ populations were selected through the modified bulk method, and in SS-2018 the same method was continued where eight segregating $F_{4}$ populations were obtained with plants from 1.30 to $1.65 \mathrm{~m}$ in height, suitable for mechanized harvesting compact panicles, with greater synchrony in flowering, which resulted in a higher grain weight per panicle (80\%). Through the improvement of amaranth by genetic recombination, the generation of modern varieties suitable for mechanized harvesting, with profitability and sustainability of the crop are feasible.

Index words: Amaranthus spp., genetic improvement, intervarietal crosses.

\section{INTRODUCCIÓN}

La superficie sembrada en México con amaranto y su volumen de producción son bajos, con apenas 2905 ha sembradas y 8166 t de grano cosechadas en 2020 (SIAP, 2021) debido, entre otros factores, a que los ecotipos y variedades disponibles actualmente no son aptas para la cosecha mecanizada, ya que poseen caracteres indeseables como poca homogeneidad en el crecimiento, con plantas altas de hasta $2.0 \mathrm{~m}$, que propician el acame, madurez indeterminada y dehiscencia del glomérulo; estos factores dan lugar a bajos rendimientos y a una cosecha manual, que es ardua y poco rentable, pues presentan bajo índice de cosecha porque sus panojas producen reducida cantidad de grano y alta proporción de paja. Estas características contribuyen al desinterés de los productores por el cultivo, lo que ha condicionado que la superficie sembrada y el volumen de producción sea insuficiente para cubrir la demanda de este grano, por lo que las nuevas variedades deben tener menores costos de producción, ser homogéneas en porte y maduración, sin dehiscencia del glomérulo, de ciclo intermedio, con un mayor balance entre el número de florecillas pistiladas y estaminadas, que mejoren el rendimiento a través de un mayor número de granos por panoja, buena calidad de 
reventado de grano y resistentes a enfermedades (Vaidya y Jain, 1987).

Para la generación de nuevas variedades que reúnan los caracteres de referencia, es necesario complementar los métodos de selección masal que se han aplicado en este cultivo, ampliando de forma dirigida la variabilidad genética con cruzamientos interespecíficos entre progenitores de A. hypochondriacus, A. cruentus y otras especies afines (Mwase et al., 2014), que deriven en poblaciones y para su manejo mediante el método masivo modificado en generaciones tempranas $\left(F_{2}-F_{4}\right)$ y selección genealógica en generaciones avanzadas $\left(F_{5}-F_{n}\right)$, y así obtener líneas sobresalientes.

Los métodos de mejora genética que en México se han aplicado en amaranto son el de selección masal simple, que ha sido practicado durante miles de años y es tan sencillo como eliminar plantas indeseables o seleccionar las mejores dentro de una población, así como el de selección masal estratificada, semejante al de selección masal simple, pero dividido en parcelas o lotes (Espitia et al., 2012). Con estos métodos se han modificado características fenotípicas de las plantas pero no se ha contribuido mucho al cambio genético; por ello, cuando estas variedades se cultivan en ambientes fuera de las áreas donde se desarrollaron, por lo general modifican su fenotipo, principalmente su ciclo vegetativo, altura de la planta que propicia el acame, presencia de ramificaciones de hábito indeterminado y dehiscencia del glomérulo que causa el desgrane, e irregularidad en la maduración del grano. Estas características repercuten en que el rendimiento sea menor al reportado en sus áreas de adaptación.

El mejoramiento del amaranto por recombinación genética tiene el objetivo de generar variedades mediante la integración de caracteres favorables en un solo genotipo. En la $F_{1}$ de cruzamientos interespecíficos entre $A$. cruentus y A. hypochondriacus se ha encontrado heterosis, pero este fenómeno aparentemente no existe en cruzas intraespecíficas (Lehman et al, 1991; Mujica et al., 1997).

El género Amaranthus se caracteriza por su gran diversidad genética, lo que permite a los investigadores realizar estudios de taxonomía y nomenclatura para la caracterización del germoplasma (De la $\mathrm{O}$ et al., 2012; Ruiz et al., 2013); también facilita el estudio de su evolución en diferentes nichos ecológicos, por lo que permite detectar fuentes de resistencia a plagas y enfermedades $u$ otras características agronómicas de interés para los productores, industriales y consumidores, caracteres que pueden aprovecharse para incorporarlos en otras variedades a través de cruzamientos (Brenner et al., 2000). Así, en un mediano plazo a través de nuevas técnicas de cruzamientos, seguidas por la combinación de manejo poblacional en generaciones tempranas por el método masivo modificado y selección genealógica en generaciones avanzadas, es posible generar variedades con características adecuadas a las condiciones climáticas y edáficas de una determinada región o tipo de agricultura que se requiera, ya sea tradicional o tecnificada (Alejandre et al., 2012). Sin duda, un aspecto que por muchos años había frenado el mejoramiento por recombinación de progenitores fue el desconocimiento de la técnica para la polinización artificial.

Por todo lo anterior, el objetivo de esta investigación fue la obtención de líneas avanzadas $F_{4}$ obtenidas de cruzamientos simples $(A \times B)$ para avanzar a una fase superior y contar con líneas promisorias para variedades adecuadas a los productores de amaranto.

\section{MATERIALES Y MÉTODOS}

\section{Selección de progenitores}

Se utilizaron materiales del Banco de Germoplasma de Amaranto del Instituto Nacional de Investigaciones Forestales, Agrícolas y Pecuarias (INIFAP) y colectas de cultivares nativos del estado de Morelos (Cuadro 1), los cuales fueron sembrados durante el ciclo primaveraverano 2013 y 2014 en el Campo Experimental de Zacatepec, Morelos, México.

Los progenitores $1,6,7,8,10,12$ y 14 procedieron del estado de Morelos, México; 2 y 4 de los Estados Unidos de América; el 3 de los Himalayas, Nepal; el 5 de Europa, y 9, 11 y 13 de los Valles Altos de México.

\section{Obtención y manejo de semillas $\mathrm{F}_{0}$}

Los cultivares seleccionados como progenitores masculinos se establecieron en condiciones de campo para la producción y recolección de polen, mientras que las variedades Revancha y Criollo de Amilcingo, que fueron usados tanto como progenitores femeninos como masculinos, se sembraron en charolas, al igual que los genotipos A. hybridus K 593 y Amaranthus sp. K340 de República Checa (Cuadro 2).

\section{Establecimiento del lote de progenitores masculinos en el campo}

Los progenitores masculinos se establecieron en campo para que experimentaran un desarrollo normal y buena producción de polen en los cruzamientos con las mini-plantas hembras (metodología desarrollada en 
Cuadro 1. Características agronómicas de genotipos de amaranto seleccionados como progenitores en el programa de cruzamientos. Campo Experimental Zacatepec, Zacatepec, Morelos, México, PV-2013/2014.

\begin{tabular}{llllll}
\hline No. & Progenitores & AP & CVE & TIP y COLP & PMIL \\
\hline 1 & A. cruentus var. Morelos & 1.10 & 93 & Abierta, verde & 0.882 \\
2 & A. cruentus var. Amont USA & 1.30 & 89 & Intermedia verde & 0.863 \\
3 & A. hypochondriacus Nepal 2255 Rojo & 1.00 & 93 & Abierta, guinda & 0.648 \\
4 & A. hybridus K593 & 0.95 & 89 & Abierta, púrpura & 0.690 \\
5 & Amaranthus sp. K340 Rep. Checa & 0.90 & 89 & Abierta, púrpura & 0.704 \\
6 & A. cruentus CV Don Guiem & 1.60 & 98 & Compacta, verde & 0.889 \\
7 & SL-2010 MOR 44 & 1.40 & 99 & Abierta guinda & 0.872 \\
8 & Naranja o canela, Mario Vidal Caporal & 2.18 & 106 & Intermedia amarilla & 0.936 \\
9 & Sel. Revancha panoja verde, "Grano oro", & 1.85 & 106 & Compacta, verde & 0.912 \\
10 & Aleazar Cerezo & 1.80 & 106 & Compacta verde & 0.952 \\
11 & Nutrisol & 1.50 & 89 & Abierta púrpura & 0.543 \\
12 & Criollo de Amilcingo & 2.17 & 96 & Intermedia amarilla & 0.925 \\
13 & Revancha & 2.10 & 93 & Compacta verde & 0.954 \\
14 & Payasa & 2.20 & 106 & Intermedia púrpura/verde/rosa/ & 0.927 \\
\hline
\end{tabular}

AP. altura de planta (m), CVE: ciclo vegetativo (días), TIP. tipo panoja, COLP. color de panoja, PMIL: peso de mil semillas (g).

la época de los 1980s por el grupo de investigadores de Rodale Research Center, mencionado por Espitia et al., 2010); se realizaron dos fechas de siembra con diferencia de 10 días para disponer de polen. Cada genotipo se aisló con una barrera física (surcos con maíz). Para la obtención de mini-plántulas de los progenitores femeninos, la siembra se efectuó en charolas en condiciones de invernadero en sustrato de cascarilla de arroz $50 \%$ + cachaza $50 \%$.

\section{Proceso de emasculación y polinización}

Las plántulas de progenitores femeninos fueron trasplantadas a los 18 días en macetas de terracota de 20.3 $\mathrm{cm}$, donde se pudieron desarrollar plantas con glomérulos de florecillas pistiladas y estaminadas. Las florecillas estaminadas fueron cuidadosamente emasculadas por las mañanas entre las 7:00 y 9:00 h. En el campo se obtuvo el polen de tres a cinco inflorescencias para realizar la polinización de las florecillas femeninas, lo que se realizó entre las 9:00 y las 11:00 h. Las semillas $F_{1}$ cosechadas de cada genotipo se conservaron en un cuarto frío.

\section{Obtención y manejo de $F_{1}$}

Se realizaron 68 cruzamientos con amarre de 30 a 50 $\%$, obteniendo un total de 6402 semillas $F_{1}$, con las que se formaron 19 grupos.

Las 19 nuevas poblaciones $F_{1}$ se sembraron en invernadero y posteriormente se trasplantaron a campo, donde cada población se aisló físicamente con una barrera de maíz, posteriormente la cosecha se efectuó de forma masiva en cada caso para obtener la semilla $F_{2}$.

\section{Manejo de las poblaciones $F_{2}$ para obtención de la generación $\mathrm{F}_{3}$}

El manejo de las primeras poblaciones segregantes se realizó en el ciclo P/V-2017, de las que se obtuvieron las poblaciones $F_{3}$ La siembra se realizó en nueve surcos de $80 \mathrm{~m}$ de largo, en forma manual, en el lomo del surco con dos a tres semillas cada $10 \mathrm{~cm}$. Posterior a la emergencia, se dejó una plántula cada $10 \mathrm{~cm}$ para selección por su morfología, respuesta a 
Cuadro 2. Semilla obtenida de los 19 grupos $F_{1}$ de amaranto generado a través de cruzas manuales. Campo Experimental de Zacatepec, Morelos, México, ciclo I/P-2014.

\begin{tabular}{lcc}
\hline Grupo & \multicolumn{1}{c}{ Progenitores } & \\
\cline { 2 - 3 } Zac-1-2014 & Femenino & Masculino \\
Zac-2-2014 & A. hybridus K593 (roja) & Criollo Amilcingo \\
Zac-3-2014 & Criollo Amilcingo (amarilla) & A. hybridus K593 (purpura) \\
Zac-4-2014 & A. cruentus var. Amont USA (verde) & Nutrisol (púrpura) \\
Zac-5-2014 & SL-2010-MOR-43 (amarilla) & A. hybridus K593 (púrpura) \\
Zac-6-2014 & A. cruentus Mexicano INTA (verde) & A. hybridus K593 (púrpura) \\
Zac-7-2014 & SL-2010-MOR-41 (amarilla) & A. hybridus K593 (púrpura) \\
Zac-8-2014 & Amarilla vara dura (verde) & A. hybridus K593 (púrpura) \\
Zac-9-2014 & Criollo de Amilcingo PV-2012 (amarilla) & A. hybridus K593 (púrpura) \\
Zac-10-2014 & Revancha (verde) & A. hybridus K593 (púrpura) \\
Zac-11-2014 & A. hybridus K593 (púrpura) & Revancha (verde) \\
Zac-12-2014 & A. hypochondriacus 2255 Nepal (guinda) & Criollo de Amilcingo (amarilla) \\
Zac-13-2014 & Criollo de Amilcingo (amarilla) & Amaranthus sp. K340 \\
Zac-14-2014 & Rep Checa (guinda) \\
Zac-15-2014 & A. cruentus var. Morelos (guinda) & Criollo de Amilcingo \\
Zac-16-2014 & A. cruentus 93 CV Don León (verde) & SL-2010 MOR-44 (púrpura) \\
Zac-17-2014 & A. cruentus CV Don Guiem (verde) & Amarilla Vara dura. Temoac (verde) \\
Zac-18-2014 & Naranja o canela. Mario Vidal Caporal (amarilla) & SL-2010 MOR-44 (púrpura) \\
Zac-19-2014 & Sel. Revancha panoja verde, grano oro (verde) & Nutrisol (púrpura) \\
\hline
\end{tabular}

plagas y enfermedades, además de los caracteres de inflorescencias, granos y rendimiento (CP-SNICS, 2006).

En madurez del grano se realizaron actividades inherentes al método masivo modificado, descartando plantas enfermas o con características indeseables, crecimiento irregular de plantas o de sus inflorescencias al desgrane; seleccionando aquellas con inflorescencias sanas y buenas proporciones de florecillas femeninas versus masculinas, para lograr una tasa de polinización aceptable; selección de las plantas con altura intermedia y resistencia al acame, con ciclo vegetativo de precoz a intermedio, con regularidad de las ramificaciones de las plantas y panojas, con aceptable producción de grano, tamaño e indehisciencia de éste, descartando inflorescencias con altos volúmenes de tamo o basura. De las plantas preseleccionadas en cada población en madurez del grano se efectuó el corte de las panojas en forma individual. Se pesaron los granos de cada panoja; al final se mezcló el grano correspondiente de las plantas seleccionadas por población, dando como resultado la obtención de las poblaciones $F_{3}$ a través de método masivo modificado.

En cada una de poblaciones obtenidas en la $\mathrm{F}_{4}$ fue realizada la selección de plantas sanas con adecuado fenotipo morfológico, consistente en altura intermedia y buen tipo de planta, con resistencia al acame, ciclo vegetativo de precoz a intermedio, regularidad de las ramificaciones de las plantas y de las panojas, aceptable producción de grano de buen tipo, tamaño e indehisciencia para resistencia al desgrane, descartándose aquellas plantas en cuyos inflorescencias se detectaron altos volúmenes de tamo debido a la baja tasa de polinización.

\section{Análisis de la información}

Se obtuvieron estadísticas descriptivas de cada 
población para los caracteres cuantitativos altura de la planta (AP, m), grosor de tallo $(\mathrm{GT}, \mathrm{cm})$, longitud de la panoja (LPA, cm) y porcentaje de grano; y los cualitativos obtenidos de acuerdo con la guía de la UPOV (2008), tales como color de tallo y color de panoja. Posteriormente, se realizó una comparación entre las poblaciones obtenidas en $F_{3}$ con las obtenidas en $F_{4}$ para porcentaje total de grano.

\section{RESULTADOS Y DISCUSIÓN}

De las 19 poblaciones segregantes $F_{2}$, las poblaciones ZAC-14-14 y ZAC-19-14 fueron descartadas por presentar baja aptitud combinatoria de sus progenitores, y características indeseables como alta incidencia de enfermedades. De las 17 poblaciones restantes se obtuvo la generación $\mathrm{F}_{3}$ (Cuadro 3), por lo que el método de selección está en función de los objetivos del mejoramiento (EspitiaRangel, 1994), las características del material de inicio y de los recursos disponibles.

En la variable de peso de semillas, las panojas produjeron diferentes cantidades de semillas, donde destacaron las poblaciones 1, 2, 3, 4, 6, 10 y 14, ya que produjeron mayores pesos de grano que de tamo, aunque en las poblaciones restantes sucedió lo contrario, pero sus panojas correspondieron a plantas con altura intermedia, de ciclo precoz y buen tamaño de panojas, por ello no se descartaron, esperando que en la siguiente generación se presente posible segregación para mayor peso de grano (Cuadro 3).

\section{Obtención de poblaciones $\mathrm{F}_{4}$}

De las 17 poblaciones segregantes $F_{3^{\prime}}$ sembradas en 2017, 10 poblaciones fueron descartadas por su notable segregación hacia características indeseables e incidencia de diferentes enfermedades, por lo que de las siete poblaciones restantes se obtuvo la generación $\mathrm{F}_{4}$ por sus características agronómicas deseables. Aunque el porte de algunas plantas sobrepasó el límite del ideotipo deseado $(1.6 \mathrm{~m})$, presentaron tallos gruesos o panojas deseables (compactas y con buena cantidad de grano). Para ello, se seleccionaron plantas de las poblaciones 1, 7, $8,9,10,11$ y 13 . Es notable que en la mayoría de las plantas de las poblaciones $\mathrm{F}_{4}$ se registró un aumento significativo del porcentaje de grano, en comparación con los granos obtenidos en las poblaciones $F_{3}$ en 2017.

En el Cuadro 4 se muestran las características de la población ZAC-1-14-0ma-58B-16B $\left(F_{4}\right)$, de la cual se seleccionaron 16 plantas. Se presentaron tallos delgados, en su mayoría rayados, firmes y resistentes al acame, con alturas de 1.2 a $1.7 \mathrm{~m}$, panojas compactas y de buena longitud (40 a $65 \mathrm{~cm}$ ), la mayoría de color vino. El mayor porcentaje de grano fue de $85.9 \%$ y el menor de $50.5 \%$.

De la población ZAC-7-14-0ma-10B-8B $\left(F_{4}\right)$ se seleccionaron ocho plantas, como se presenta en el Cuadro 5 , las plantas presentaron alturas de 1.4 a $1.6 \mathrm{~m}$, todas con tallos rayados, con panojas compactas, en su mayoría de color vino. El mayor porcentaje de grano fue de 85.0 \% y el menor de $50 \%$, lo cual concuerda con lo mencionado

Cuadro 3. Poblaciones segregantes $\mathrm{F}_{3}$ obtenidas a través del método de selección masivo modificado, Campo Experimental Zacatepec, ciclo PV-2017.

\begin{tabular}{llllllll}
\hline Pob. & Genealogía & DM & PS (g) & Pob & Genealogía & DM & PS (g) \\
\hline 1 & ZAC-1-14-0ma-58B & $76-98$ & 707.7 & 10 & ZAC-10-14-0ma-42B & $76-98$ & 339.7 \\
2 & ZAC-2-14-0ma-15B & 82 & 105.1 & 11 & ZAC-11-14-0ma-21B & 102 & 19.5 \\
3 & ZAC-3-14-0ma-20B & 97 & 77.82 & 12 & ZAC-12-14-0ma-21B & 97 & 40.1 \\
4 & ZAC-4-14-0ma-40B & 82 & 179.9 & 13 & ZAC-13-14-0ma-20B & 102 & 16.9 \\
5 & ZAC-5-14-0ma-20B & 97 & 38.4 & 14 & ZAC-15-14-0ma-31B & 82 & 230.8 \\
6 & ZAC-6-14-0ma-29B & 82 & 201.4 & 15 & ZAC-16-14-0ma-19B & 102 & 29.7 \\
7 & ZAC-7-14-0ma-10B & 94 & 19.6 & 16 & ZAC-17-14-0ma-19B & 102 & 38.3 \\
8 & ZAC-8-14-0ma-11B & 94 & 23.2 & 17 & ZAC-18-14-0ma-10B & 105 & 20.7 \\
9 & ZAC-9-14-0 ma-14B & 94 & 53.5 & & & &
\end{tabular}

Pob: número de población, DM: días a madurez, PS: peso de semilla. 
por Weber y Kauffman (1990; Com.Pers.) ${ }^{1}$ sobre los requerimientos de ideotipos con mayor porcentaje de grano.

Las características de la población Zac-8-14-0ma-14B28B $\left(F_{4}\right)$, se muestran en el Cuadro 6. La altura de planta fluctuó de 1.1 a $1.6 \mathrm{~m}$, con tallos rayados con resistencia al acame con grosor mediano en su mayoría y el resto grueso. Las panojas fueron compactas, con longitudes de 45 a $65 \mathrm{~cm}$, de las cuales 21 presentaron color rosa y el resto color vino. El mayor porcentaje de grano fue de 87.5 $\%$ y el menor de $70 \%$.

Las características de la población ZAC-9-14-0ma14B-26B $\left(F_{4}\right)$ se muestran en el Cuadro 7. La altura de planta fluctuó de 1.4 a $1.6 \mathrm{~m}$, la mayoría de tallos fueron de color amarillos y otros presentaron rayas, ocho de grosor delgado y 18 de grosor mediano. La longitud de panoja osciló de 45 a $70 \mathrm{~cm}$, de las cuales 10 fueron de tono rosa, 11 verde y cinco de color vino. El mayor

\footnotetext{
'Weber F. L and C. S. Kauffman (1990) Plant breeding and seed production. In: Proceedings of the 4th National Amaranth Symposium: Perspectives on Production, Processing and Marketing. American Amaranth Institute. Minneapolis, Minnesota, USA. p:115.
}

porcentaje de grano fue de 83.2 y el menor de $64.9 \%$.

Las características de la población ZAC-10-14-0ma42B-23B $\quad\left(F_{4}\right)$ se observan en el Cuadro 8 , donde se obtuvieron alturas de planta de 1.3 a $1.6 \mathrm{~m}$, cinco plantas presentaron tallos lisos y el resto fueron tallos rayados, 20 plantas con grosor mediano, dos delgados y uno grueso. Las panojas compactas, con longitudes de 40 a $55 \mathrm{~cm}, 12$ de color vino, cuatro verdes y siete de color rosa.

En el Cuadro 9 se muestran las variables de la población $\mathrm{F}_{4}$ ZAC-11-14-0ma-21B-14B, 14 plantas fueron seleccionadas por su altura de 1.4 a $1.60 \mathrm{~m}$, de las cuales seis tuvieron tallos rayados y ocho lisos, la mayoría fueron gruesos. Panojas compactas de 40 a $80 \mathrm{~cm}$ de longitud, 11 mostraron color verde y tres color vino, con un porcentaje de grano mayor de $81.6 \%$.

En el Cuadro 10 se muestran las variables de la población $\mathrm{F}_{4}$ ZAC-13-14-0ma-20B-6B (6 plantas seleccionadas), las que presentaron alturas de 1.5 a $1.6 \mathrm{~m}$, cinco mostraron tallos rayados y uno liso, con grosor de tallo en su mayoría mediano. Las panojas fueron compactas (Ruiz et al., 2018), de longitudes de $45 \mathrm{a} 65 \mathrm{~cm}$ y todas de color rosa, con el

Cuadro 4. Características de las plantas seleccionadas en la población ZAC-1-14-0ma-58B-16B $\left(\mathrm{F}_{4}\right)$, ciclo P/V-2018, Zacatepec, Morelos.

\begin{tabular}{lllllll}
\hline Planta & AP & CT & GT & LPA & CPA & GR \\
\hline 1 & 1.2 & Amarillo/ rayas guinda & Delgado & 40 & Vino & 71.2 \\
2 & 1.4 & Amarillo/ rayas guinda & Mediano & 57 & Vino & 51.1 \\
3 & 1.3 & Verde/ rayas guinda & Delgado & 55 & Vino & 76.7 \\
4 & 1.4 & Amarillo/ rayas guinda & Delgado & 55 & Vino & 68.5 \\
5 & 1.2 & Amarillo/ rayas guinda & Delgado & 55 & Vino & 76.7 \\
6 & 1.4 & Amarillo/ rayas guinda & Delgado & 65 & Vino & 80.6 \\
7 & 1.4 & Amarillo/ rayas guinda & Delgado & 55 & Vino & 72.8 \\
8 & 1.2 & Amarillo/ rayas guinda & Delgado & 56 & Vino & 85.9 \\
9 & 1.3 & Amarillo/ rayas guinda & Delgado & 50 & vino & 70.2 \\
10 & 1.2 & Amarillo/ rayas guinda & Delgado & 42 & Vino & 74.5 \\
11 & 1.2 & Amarillo/ rayas guinda & Delgado & 58 & Vino & 81.8 \\
12 & 1.4 & Amarillo/ rayas guinda & Delgado & 65 & Vino & 78.8 \\
13 & 1.2 & Amarillo/rayas guinda & Delgado & 44 & Vino & 74.5 \\
14 & 1.3 & Amarillo/ rayas guinda & Delgado & 50 & Vino & 82.4 \\
15 & 1.7 & Verde & Delgado & 60 & Verde & 77.7 \\
16 & 1.4 & Verde & Delgado & 57 & Verde & 50.5 \\
\hline
\end{tabular}

AP. altura de planta (m), CT: color de tallo, GT: grosor de tallo, LPA: longitud de panoja (cm), CPA: color de panoja, GR: grano (\%). 
Cuadro 5. Características de las plantas seleccionadas en la población ZAC-7-14-0ma-10B-8B $\left(F_{4}\right)$, ciclo P/V-2018, Zacatepec, Morelos.

\begin{tabular}{|c|c|c|c|c|c|c|}
\hline Planta & $\mathrm{AP}$ & CT & GT & LPA & CPA & GR \\
\hline 1 & 1.6 & Amarillo con rayas guinda & Mediano & 60 & vino & 50 \\
\hline 2 & 1.5 & Amarillo con rayas guinda & Delgado & 55 & Vino & 85.0 \\
\hline 3 & 1.6 & Amarillo con rayas guinda & Delgado & 55 & Vino & 84.3 \\
\hline 4 & 1.5 & Amarillo con rayas guinda & Delgado & 60 & Vino & 84.1 \\
\hline 5 & 1.5 & Amarillo con rayas guinda & Mediano & 64 & Vino & 81.7 \\
\hline 6 & 1.4 & Amarillo con rayas guinda & Mediano & 70 & Vino & 73.1 \\
\hline 7 & 1.6 & Amarillo con rayas guinda & Mediano & 67 & Vino & 81.6 \\
\hline 8 & 1.5 & Amarillo con rayas guinda & Mediano & 55 & Rosa & 72.5 \\
\hline
\end{tabular}

AP. altura de planta (m), CT: color de tallo, GT: grosor de tallo, LPA: longitud de panoja (cm), CPA: color de panoja, GR: grano (\%).

mayor porcentaje de grano de $80.7 \%$ y $71.1 \%$ el menor.

La comparación del comportamiento de las poblaciones segregantes seleccionadas $\mathrm{F}_{3}$ y $\mathrm{F}_{4}$ se puede observar en la Figura 1. En la población $F_{3}$ se seleccionaron 409 plantas, cuya producción de grano fue menor al 50 \%; en cambio, en la población $\mathrm{F}_{4}$ se cosecharon 122 plantas, aunque el número fue menor a la población anterior, la producción de grano aumentó considerablemente del 60 al $80 \%$.

Con respecto al ciclo vegetativo, en la población $\mathrm{F}_{3}$ los días a madurez oscilaron en un intervalo de 78 a 102 días, mientras que las plantas de las poblaciones $\mathrm{F}_{4}$ presentaron mayor precocidad, ya que presentaron un periodo de 78 a 92 días a la madurez. Para las condiciones del estado de Morelos, México se requiere de este tipo de genotipos, ya que el ciclo regular de lluvias que se registra es de junio a septiembre.

De acuerdo con los resultados obtenidos, con el manejo poblacional $\left(\mathrm{F}_{2}-\mathrm{F}_{4}\right)$, en la última generación se puede considerar que se han seleccionado plantas con un resultado favorable para la obtención de variedades modernas (Tavitas et al., 2015), ya que se obtuvieron plantas con alturas de entre 1.30 y $1.65 \mathrm{~m}$, aptas para la cosecha mecanizada, con un mejor balance de florecillas pistiladas versus estaminadas en sus inflorescencias, lo que se tradujo en una tasa mayor de polinización, y por lo tanto, un mayor volumen y peso de grano, lo cual coincide con lo encontrado por Maughan et al. (2011). Estos avances indican que a través de la mejora genética del amaranto por recombinación genética dirigida, es factible la generación de variedades modernas con ideotipos aptos para cosecha mecanizada, con un mayor equilibrio entre florecillas pistiladas versus estaminadas en sus inflorescencias (Kulakow y Jain,1987) y con mayor potencial de rendimiento con respecto a las variedades actuales (Kauffman, 1990).

Como procedimiento global, puede mencionarse que de los 65 cruzamientos simples $(A \times B)$ efectuados en 2013, se obtuvieron 19 grupos con un total de 6402 semillas $F_{0}$. De las 19 poblaciones $F_{2}$ sembradas en 2017, dos de ellas fueron descartadas totalmente por baja aptitud combinatoria de sus progenitores. De las 17 poblaciones restantes se obtuvo la generación $F_{3^{\prime}}$ constituida por 409 plantas De las 18 poblaciones $F_{3}$ manejadas en 2018 fueron descartadas 12 de éstas, y de las siete poblaciones vigentes sólo se obtuvieron 122 plantas en la generación $\left(F_{4}\right)$.

\section{CONCLUSIONES}

La hibridación es un método efectivo de mejoramiento genético en amaranto, debido al aprovechamiento de la variabilidad genética disponible en forma natural y constituye el primer ensayo en México sobre éste método de mejora. En $\mathrm{F}_{4}$ se obtuvieron líneas avanzadas de amaranto con alturas de 1.45 a $1.60 \mathrm{~m}$, con tallos resistentes al acame lo que permite la cosecha mecanizada, aprovechando la variabilidad generada mediante recombinación genética a través de la hibridación. En la $\mathrm{F}_{4}$ se obtuvieron mayores porcentajes de grano de entre 60 y $80 \%$. 
Cuadro 6. Características de las plantas seleccionadas en la población ZAC-8-14-0ma-14B-28B $\left(\mathrm{F}_{4}\right)$, ciclo P/V-2018, Zacatepec, Morelos.

\begin{tabular}{|c|c|c|c|c|c|c|}
\hline Planta & AP & $\mathrm{CT}$ & $\mathrm{GT}$ & LPA & CPA & $\mathrm{GR}$ \\
\hline 1 & 1.2 & Amarillo/ rayas guinda & Mediano & 50 & Rosa & 82.6 \\
\hline 2 & 1.3 & Amarillo/ rayas guinda & Mediano & 45 & Rosa & 83.6 \\
\hline 3 & 1.2 & Amarillo/ rayas guinda & Mediano & 45 & Rosa & 84.3 \\
\hline 4 & 1.4 & Amarillo/ rayas rosa & Mediano & 60 & Rosa & 82.7 \\
\hline 5 & 1.4 & Amarillo/ rayas guinda & Mediano & 65 & Rosa & 82.3 \\
\hline 6 & 1.4 & Amarillo/ rayas guinda & Mediano & 60 & Rosa & 81.8 \\
\hline 7 & 1.2 & Amarillo/ rayas guinda & Mediano & 50 & Rosa & 83.9 \\
\hline 8 & 1.4 & Amarillo/ rayas guinda & Mediano & 52 & Rosa & 84.8 \\
\hline 9 & 1.5 & Amarillo/ rayas guinda & Mediano & 62 & Rosa & 79.2 \\
\hline 10 & 1.5 & Amarillo/ rayas guinda & Grueso & 60 & Rosa & 83.5 \\
\hline 11 & 1.2 & Amarillo/ rayas guinda & Mediano & 52 & Rosa & 83.4 \\
\hline 12 & 1.42 & Amarillo/ rayas guinda & Mediano & 55 & Rosa & 82.1 \\
\hline 13 & 1.2 & Amarillo/ rayas guinda & Mediano & 50 & Rosa & 71.7 \\
\hline 14 & 1.3 & Amarillo/ rayas guinda & Mediano & 50 & Rosa & 82.7 \\
\hline 15 & 1.2 & Amarillo/ rayas guinda & Mediano & 50 & Rosa & 74.9 \\
\hline 16 & 1.3 & Amarillo/ rayas guinda & Delgado & 60 & Rosa & 74.3 \\
\hline 17 & 1.6 & Amarillo/ rayas guinda & Grueso & 65 & Rosa & 82.9 \\
\hline 18 & 1.1 & Amarillo/ rayas guinda & Mediano & 45 & Rosa & 87.5 \\
\hline 19 & 1.3 & Amarillo/ rayas guinda & Mediano & 45 & Rosa & 81.8 \\
\hline 20 & 1.3 & Amarillo/ rayas rosa & Mediano & 50 & Rosa & 82.8 \\
\hline 21 & 1.3 & Amarillo/ rayas guinda & Mediano & 45 & Rosa & 84.3 \\
\hline 22 & 1.4 & Guinda/ rayas amarillo & Mediano & 55 & Vino & 82.7 \\
\hline 23 & 1.3 & Amarillo/ rayas guinda & Delgado & 45 & Vino & 83.2 \\
\hline 24 & 1.3 & Amarillo/ rayas guinda & Grueso & 55 & Vino & 82.9 \\
\hline 25 & 1.4 & Amarillo/ rayas guinda & Mediano & 50 & Vino & 83.2 \\
\hline 26 & 1.5 & Amarillo/ rayas guinda & Mediano & 60 & Vino & 80.1 \\
\hline 27 & 1.5 & Amarillo/ rayas guinda & Mediano & 60 & Vino & 81.1 \\
\hline 28 & 1.4 & Amarillo/rayas guinda & Mediano & 55 & Vino & 70.0 \\
\hline
\end{tabular}

AP. altura de planta (m), CT: color de tallo, GT: grosor de tallo, LPA: Iongitud de panoja (cm), CPA: color de panoja, GR: grano (\%). 
Cuadro 7. Características de las plantas seleccionadas en la población ZAC-9-14-0ma-14B-26B $\left(F_{4}\right)$, ciclo P/V-2018, Zacatepec, Morelos.

\begin{tabular}{|c|c|c|c|c|c|c|}
\hline Planta & $\mathrm{AP}$ & $\mathrm{CT}$ & GT & LPA & CPA & $\mathrm{GR}$ \\
\hline 1 & 1.6 & Amarillo & Delgado & 57 & Rosa & 83.2 \\
\hline 2 & 1.5 & Verde & Mediano & 55 & Rosa & 64.9 \\
\hline 3 & 1.5 & Amarillo & Delgado & 50 & Rosa & 78.1 \\
\hline 4 & 1.5 & Amarillo & Delgado & 45 & Verde & 81.7 \\
\hline 5 & 1.6 & Verde pálido & Mediano & 70 & Verde & 78.5 \\
\hline 6 & 1.6 & Verde pálido & Mediano & 57 & Verde & 80.4 \\
\hline 7 & 1.5 & Amarillo & Delgado & 55 & Rosa & 79.9 \\
\hline 8 & 1.6 & Amarillo & Mediano & 50 & Rosa & 83.2 \\
\hline 9 & 1.4 & Amarillo & Delgado & 55 & Rosa & 78.9 \\
\hline 10 & 1.5 & Amarillo & Delgado & 45 & Verde & 80.7 \\
\hline 11 & 1.5 & Amarillo & Mediano & 65 & Rosa & 71.4 \\
\hline 12 & 1.5 & Amarillo & Mediano & 45 & Verde & 77.2 \\
\hline 13 & 1.6 & $\begin{array}{c}\text { Amarillo con rayas } \\
\text { guindas }\end{array}$ & Mediano & 50 & Vino & 82.3 \\
\hline 14 & 1.6 & Amarillo & Mediano & 55 & Verde & 82.5 \\
\hline 15 & 1.5 & Amarillo & Mediano & 55 & Verde & 78.4 \\
\hline 16 & 1.4 & Amarillo & Mediano & 55 & Vino & 71.2 \\
\hline 17 & 1.6 & Amarilla & Mediano & 55 & Verde & 73.3 \\
\hline 18 & 1.5 & $\begin{array}{c}\text { Amarillo con rayas } \\
\text { guindas }\end{array}$ & Mediano & 62 & Vino & 81.1 \\
\hline 19 & 1.6 & Amarillo & Mediano & 50 & Verde & 83.1 \\
\hline 20 & 1.4 & $\begin{array}{c}\text { Amarillo con rayas } \\
\text { guinda }\end{array}$ & Delgado & 45 & Rosa & 77.9 \\
\hline 21 & 1.6 & Amarillo & Mediano & 45 & Verde & 70.8 \\
\hline 22 & 1.6 & Amarillo & Delgado & 45 & Verde & 76.6 \\
\hline 23 & 1.4 & $\begin{array}{c}\text { Amarillo con rayas } \\
\text { guinda }\end{array}$ & Mediano & 45 & Rosa & 64.6 \\
\hline 24 & 1.5 & $\begin{array}{c}\text { Amarillo con rayas } \\
\text { guinda }\end{array}$ & Mediano & 55 & Rosa & 73.1 \\
\hline 25 & 1.5 & $\begin{array}{c}\text { Amarillo con rayas } \\
\text { guinda }\end{array}$ & Mediano & 50 & Vino & 72.2 \\
\hline 26 & 1.6 & $\begin{array}{c}\text { Amarillo con rayas } \\
\text { guinda }\end{array}$ & Mediano & 50 & Vino & 73.9 \\
\hline
\end{tabular}

AP. altura de planta (m), CT: color de tallo, GT: grosor de tallo, LPA: longitud de panoja (cm), CPA: color de panoja, GR: grano (\%). 
Cuadro 8. Características de las plantas seleccionadas en la población ZAC-10-14-0ma-42B-23B $\left(F_{4}\right)$, ciclo P/V-2018, Zacatepec, Morelos.

\begin{tabular}{|c|c|c|c|c|c|c|}
\hline Planta & $\mathrm{AP}$ & CT & GT & LPA & CPA & GR \\
\hline 1 & 1.40 & Amarillo con rayas guinda & Mediano & 55 & Vino & 74.40 \\
\hline 2 & 1.45 & Amarillo & Mediano & 45 & Verde & 71.56 \\
\hline 3 & 1.35 & Amarillo con rayas guinda & Mediano & 45 & Rosa & 75.87 \\
\hline 4 & 1.45 & Amarillo con rayas guinda & Mediano & 50 & Rosa & 68.96 \\
\hline 5 & 1.53 & Amarillo con rayas guinda & Mediano & 45 & Rosa & 71.04 \\
\hline 6 & 1.60 & Amarillo con rayas guinda & Mediano & 55 & Vino & 75.44 \\
\hline 7 & 1.50 & Amarillo con rayas guinda & Mediano & 45 & Vino & 76.23 \\
\hline 8 & 1.30 & Amarillo con rayas guinda & Mediano & 55 & Rosa & 77.43 \\
\hline 9 & 1.55 & Amarillo con rayas guinda & Mediano & 50 & Vino & 78.56 \\
\hline 10 & 1.50 & Amarillo & Mediano & 45 & Verde & 79.77 \\
\hline 11 & 1.65 & Amarillo con rayas guinda & Grueso & 50 & Vino & 73.97 \\
\hline 12 & 1.25 & Amarillo con rayas guinda & Mediano & 40 & Vino & 77.53 \\
\hline 13 & 1.55 & Amarillo con rayas guinda & Mediano & 50 & Vino & 67.22 \\
\hline 14 & 1.55 & Amarillo con rayas guinda & Mediano & 55 & Vino & 76.51 \\
\hline 15 & 1.45 & Amarillo con rayas guinda & Mediano & 50 & Vino & 76.26 \\
\hline 16 & 1.45 & Amarillo con rayas guinda & Mediano & 55 & Rosa & 77.36 \\
\hline 17 & 1.55 & Amarillo & Mediano & 50 & Rosa & 69.24 \\
\hline 18 & 1.40 & Amarillo con rayas guinda & Mediano & 40 & Vino & 75.64 \\
\hline 19 & 1.40 & Amarillo con rayas guinda & Mediano & 50 & Vino & 76.35 \\
\hline 20 & 1.45 & Amarillo con rayas guinda & Delgado & 45 & Rosa & 75.34 \\
\hline 21 & 1.50 & Amarillo & Mediano & 45 & Verde & 69.73 \\
\hline 22 & 1.30 & Amarillo & Delgado & 45 & Verde & 79.45 \\
\hline 23 & 1.60 & Amarillo con rayas guinda & Mediano & 55 & Vino & 83.59 \\
\hline
\end{tabular}

AP. altura de planta (m), CT: color de tallo, GT: grosor de tallo, LPA: longitud de panoja (cm), CPA: color de panoja, GR: grano (\%).

\section{BIBLIOGRAFÍA}

Alejandre I. G., C. G. S. Valdés L. y J. García P. (2012) Selección y adaptación de variedades criollas de amaranto (Amaranthus cruentus L.) en el Noreste de México. In: Amaranto: Ciencia y Tecnología. Libro Científico No. 2. E. Espitia R. (ed.). Instituto Nacional de Investigaciones Forestales, Agrícolas y Pecuarias. México, D. F. pp:249-256.

Brenner D. M., D. D. Baltensperger, P. A. Kulakow, J. W. Lehmann, R. L. Myers, M. M. Slabbert and B. B. Sleugh (2000) Genetic resources and breeding of Amaranthus. Plant Breeding Review 19:227-285, https://doi.org/10.1002/9780470650172.ch7
CP-SNICS, Colegio de Postgraduados-Servicio Nacional de Inspección y Certificación de Semillas (2006) Manual Gráfico para la Descripción Varietal en Amaranto (Amaranthus spp.). Colegio de Postgraduados-Servicio Nacional de Inspección y Certificación de Semillas. Montecillo, Estado de México. 43 p.

De la 0 0. M., E. Espitia R., A. V. Ayala G., J. M. Hernández C., J. L. Arellano V. y V. C. Ruiz H. (2012) Caracterización morfológica de germoplasma para grano de amaranto (Amaranthus spp.). In: Amaranto: Ciencia y Tecnología. Libro Científico No. 2. E. Espitia R. (ed.) Instituto Nacional de Investigaciones Forestales, Agrícolas y Pecuarias. México, D. F. pp:165-181.

Espitia-Rangel E. (1994) Breeding of grain amaranth. In: Amaranth Biology, Chemistry and Tecnology. O. Paredes-López (ed.). CRC 
Cuadro 9. Características de las plantas seleccionadas en la población ZAC-11-14-0ma-21B-14B $\left(F_{4}\right)$, ciclo P/V-2018, Zacatepec, Morelos.

\begin{tabular}{lllllll}
\hline Planta & AP & CT & GT & LPA & CPA & GR \\
\hline 1 & 1.6 & Amarillo & Mediano & 55 & Verde & 81.6 \\
2 & 1.5 & Amarillo & Mediano & 45 & Verde & 75.2 \\
3 & 1.6 & Amarillo & Grueso & 60 & Verde & 63.7 \\
4 & 1.5 & Amarillo & Grueso & 45 & Verde & 60.7 \\
5 & 1.5 & Amarillo/ rayas guinda & Mediano & 45 & Verde & 71.5 \\
6 & 1.5 & Amarillo/ rayas guinda & Mediano & 45 & Vino & 76.8 \\
7 & 1.6 & Verde & Grueso & 70 & Verde & 62.5 \\
8 & 1.4 & Amarillo & Mediano & 40 & Verde & 68.3 \\
9 & 1.5 & Verde/ rayas guinda & Grueso & 65 & Verde & 71.1 \\
10 & 1.4 & Amarillo/rayas guinda & Mediano & 50 & Vino & 69.4 \\
11 & 1.6 & Verde & Grueso & 65 & Verde & 67.7 \\
12 & 1.5 & Verde & Grueso & 65 & Verde & 69.2 \\
13 & 1.6 & Verde/ rayas guinda & Grueso & 75 & Verde & 77.3 \\
14 & 1.4 & Verde/ rayas guinda & Grueso & 80 & Vino & 67.5
\end{tabular}

AP. altura de planta (m), CT: color de tallo, GT: grosor de tallo, LPA: longitud de panoja (cm), CPA: color de panoja, GR: grano (\%).

Cuadro 10. Características de las plantas seleccionadas en la población ZAC-13-14-0ma-20B-6B $\left(F_{4}\right)$, ciclo P/V-2018, Zacatepec, Morelos.

\begin{tabular}{lllllll}
\hline Planta & AP & CT & GT & LPA & CPA & GR \\
\hline 1 & 1.6 & Verde con rayas guinda & Mediano & 60 & Rosa & 76.0 \\
2 & 1.6 & Amarillo con rayas guinda & Grueso & 65 & Rosa & 71.8 \\
3 & 1.5 & Amarillo con rayas guinda & Mediano & 45 & Rosa & 73.7 \\
4 & 1.5 & Vino & Mediano & 50 & Rosa & 71.7 \\
5 & 1.5 & Amarillo con rayas guinda & Mediano & 45 & Rosa & 80.7 \\
6 & 1.6 & Amarillo con rayas guinda & Mediano & 50 & Rosa & 75.1
\end{tabular}

AP. altura de planta (m), CT: color de tallo, GT: grosor de tallo, LPA: Iongitud de panoja (cm), CPA: color de panoja, GR: grano (\%).

Press. Boca Raton, Florida, USA. pp:23-38.

Espitia R. E., D. Escobedo L. y M. Aguilar D. (2012) Estrategia y metodología para el mejoramiento genético de amaranto. In: Amaranto Ciencia y Tecnología. Libro Científico No. 2. E. Espitia R. (ed.). Instituto Nacional de Investigaciones Forestales, Agrícolas y Pecuarias. México, D. F. pp:227-247.

Espitia R. E., C. Mapes S., D. Escobedo L., M. De la 0 O., P. Rivas V., G. Martínez T., L. Cortés E. y J. M. Hernández C. (2010) Conservación y Uso de los Recursos Genéticos de Amaranto en México. Centro de Investigación Regional Centro, INIFAP, Celaya, Guanajuato, México. 200 p.

Kauffman S. C. (1990) Observaciones sobre las investigaciones preliminares para el desarrollo de variedades mejoradas de amaranto de grano en cinco países. In: Amaranthus spp. Su Cultivo y Aprovechamiento. A. Trinidad S., F. Gómez L. y G Suárez R. (eds.). Colegio de Postgraduados. Montecillo, Estado de México. pp:280-288.

Kulakow P. and S. Jain (1987) Genetics of grain amaranths. 4. Variation in early generation response to selection in Amaranthus cruentus L. Theoretical and Applied Genetics 74:113-120 https://doi.org/10.1007/BF00290093

Lehman J. W., R. L. Clark and K. J. Frey (1991) Biomass heterosis and combining ability in interspecific and intraspecific rating of grain amaranths. Crop Science 31:1111-1116, https://doi. 


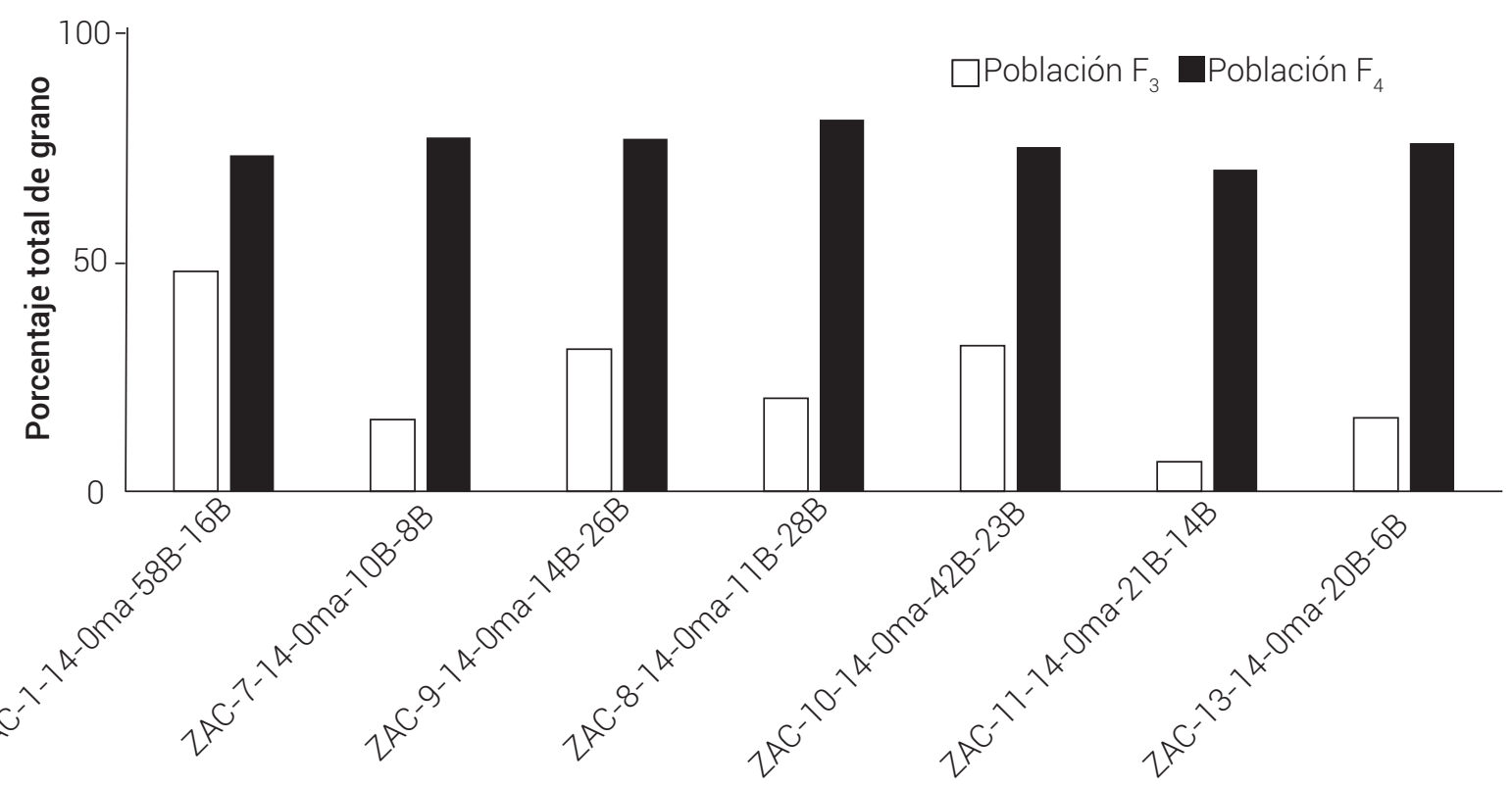

Figura 1. Porcentaje total del grano obtenido en $F_{3}$ y $F_{4}$ de las poblaciones seleccionadas.

org/10.2135/cropsci1991.0011183X003100050004x

Maughan P. J., S. M. Smith, D. J. Fairbanks and E. N. Jellen (2011) Development, characterization, and linkage mapping of single nucleotide polymorphisms in the grain amaranths (Amaranthus sp.). The Plant Genome 4:92-101, https://doi.org/10.3835/ plantgenome2010.12.0027

Mujica S. A., M. Berti D. y J. Izquierdo (1997) El Cultivo del Amaranto (Amaranthus spp.). Producción, Mejoramiento Genético y Utilización. Oficina Regional de la FAO para América Latina y el Caribe. Santiago, Chile. 145 p.

Mwase W. F., N. Kachiguma, D. Manduwa and M. F. A. Maliro (2014) Agromorphological diversity of Amaranthus species in Central Malawi. International Journal of AgriScience 4:235-241.

Ruiz H. V. C., M. De la 0 0., E. Espitia R., D. M. Sangerman-Jarquín., J. M. Hernández C. y R. Schwentesius R. (2013) Variabilidad cualitativa y cuantitativa de accesiones de amaranto determinada mediante caracterización morfológica. Revista Mexicana de Ciencias Agrícolas 4:789-801, https://doi.org/10.29312/remexca. V4i5.1176

Ruiz H. V. C., J. P. Legaria S., J. Sahagún C. y M. De la 0 0. (2018) Variabilidad genética en algunas especies cultivadas y silvestres de amaranto. Revista Mexicana de Ciencias Agrícolas 9:405-416, https://doi.org/10.29312/remexca.v9i2.1081

SIAP, Servicio de Información Agroalimentaria y Pesquera (2021) Anuario estadístico de la producción agrícola. Secretaría de Agricultura y Desarrollo Rural. Ciudad de México. https://nube.siap.gob. mx/cierreagricola/ (Noviembre 2021).

Tavitas F. L., L. Hernández A., A. Isidro 0. y R. A. Urbina R. (2015) Técnicas para la realización de cruzas en amaranto. Folleto Técnico No. 96. Campo Experimental Zacatepec, INIFAP. Zacatepec Morelos. 44 p.

UPOV, Unión Internacional para la Protección de Obtenciones Vegetales (2008) Guía para la descripción varietal en amaranto. Documento TG/247/1. Unión Internacional para la Protección de Obtenciones Vegetales. Ginebra, Suiza. $187 \mathrm{p}$

Vaidya K. R. and S. K. Jain (1987) Response to mass selection for plant height and grain yield in amaranth (Amaranthus spp). Plant Breeding 98:61-64, https://doi.org/10.1111/j.1439-0523.1987. tb01091.x 